\title{
Microphthalmia with limb anomalies
}

INSERM

\section{Source}

INSERM. (1999). Orphanet: an online rare disease and orphan drug data base.

Microphthalmia with limb anomalies. ORPHA:1106

Microphthalmia with limb anomalies, also known as ophthalmo-acromelic syndrome (OAS), is a rare developmental disorder characterized by bilateral microphthalmia or anophthalmia, synostosis, syndactyly, oligodactyly and/or polydactyly. 